\title{
Discrimination of three cyst-forming nematodes of the genus Globodera (Nema- tode: Heteroderidae) from Japan based on PCR-RFLP of ribosomal DNA
}

\author{
Taketo Uehara, ${ }^{*}$ Atsuhiko Kushida, ${ }^{\dagger}$ Kenji Itou, Takashi Narabu and Yoji Momota ${ }^{\ddagger}$ \\ Laboratory of Plant Nematology, National Agricultural Research Center for Hokkaido Region; Toyohira, Sapporo 062-8555, Japan
}

(Received 24 September 2004; Accepted 12 April 2005)

\begin{abstract}
The ITS1-5.8S-ITS2 region of rDNA sequences from three Globodera species found in Japan, G. rostochiensis, G. tabacum and G. hypolysi, were sequenced and compared. Sequence lengths for G. rostochiensis, G. tabacum and $G$. hypolysi were 1,001,1,003 and 1,016 bp, respectively. Sequence divergence within the three Globodera species ranged from 3.8 to $14.7 \%$. Eight restriction enzymes (AfaI, AluI, FspI, HhaI, HinfI, MvaI, PmaCI and SacII) were selected on the basis of sequence information. These three species were easily distinguished according to species-specific patterns by digesting the amplified products with $A l u \mathrm{I}$ or PmaCI. The FspI-digested pattern distinguished G. rostochiensis from the other Globodera species.
\end{abstract}

Key words: Globodera rostochiensis; Globodera tabacum; Globodera hypolysi; identification, RFLP

\section{INTRODUCTION}

Three species of cyst-forming nematodes of the genus Globodera have been reported in Japan. G. rostochiensis was first found in Japan in 1972, in a potato field of Makkari Village in Hokkaido (Yamada et al., 1972). It subsequently spread to other parts of Hokkaido. Infection with this species had been restricted to Hokkaido, the northernmost island of Japan, for about 20 years. However, in 1992, G. rostochiensis was found in Nagasaki Prefecture of Kyushu Island, the southern island of Japan (Nakasuga and Nakasono, 1996). In 2003, it was also found in Aomori Prefecture of Honshu, the main island of Japan. In 1976, cysts of Globodera, which are different from those of G. rostochiensis in morphological characteristics, were found in a potato growing area in Nagasaki Prefecture, and the nematode was described as G. hypolysi (Ogawa et al., 1983). Inoculation tests have proved that mugwort Artemisia princeps is a host of this species and that the potato is not a host. $G$. hypolysi was also found in the east part of Hokkaido (Yamada and Takakura, 2003). The occurrence of G. tabacum was recorded for the first time in Japan in 1998 (Sumiya et al., 2002). G. tabacum was found in a greenhouse of eggplants in Kochi Prefecture. The infected area is currently under surveillance and being subjected to control programs. Given the great importance of the potato cyst nematode ( $G$. rostochiensis) as a parasite and quarantine species, the presence of other Globodera species complicates the work of identifying cyst-forming nematodes in Japan. Identifying cystforming nematodes based on differences in morphological and morphometric characteristics requires a great deal of skill, is time-consuming and is often inconclusive for individuals. PCR-based diagnostics provide an attractive solution to these problems. Comparative analysis of coding and noncoding regions of the ribosomal DNA (rDNA) is becoming a popular tool for species and subspecies identification, and for evaluating the phylogenetic relationships among plant-parasitic nematodes from many genera (Vrain et al., 1992; Ferris et al., 1993; Iwahori et al., 1998; Orui and Mizukubo, 1999; Mizukubo et al., 2003). RFLP analysis of the internal transcribed spacers (ITS) was useful for the identification of Globodera species (Thiéry and Mugniéry, 1996; Subbotin et al., 2000). In the pres-

\footnotetext{
* To whom correspondence should be addressed at: E-mail: uehara@affrc.go.jp

${ }^{\dagger}$ Present address: National Agricultural Research Center for Hokkaido Region, Memuro, Kasai 082-0071, Japan

¥ Present address: National Agricultural Research Center, Tsukuba, Ibaraki 305-8666, Japan DOI: $10.1303 /$ aez.2005.537
} 
ent study, we performed a DNA sequencing analysis for the ITS regions of the rDNA of Japanese isolates of the three Globodera species, examined the sequence variations, and applied PCR-RFLP analysis for the discrimination of three Globodera species in Japan based on the sequences of ITS regions.

\section{MATERIALS AND METHODS}

Nematodes and DNA extraction from a single juvenile. Sixty-four isolates of $G$. rostochiensis, two isolates of $G$. hypolysi, and one isolate of $G$. tabacum were used in this study (Table 1). A cyst presoaked in sterile water was crushed and the juveniles were collected. Crude lysate of DNA was prepared using a modified method of Uehara et al. (1998). A single juvenile nematode was cut with a needle in $14 \mu \mathrm{l}$ of worm lysis buffer $(50 \mathrm{~mm} \mathrm{KCl}$, $10 \mathrm{~mm}$ Tris- $\mathrm{HCl}, \mathrm{pH} 8.0,2.5 \mathrm{~mm} \mathrm{MgCl}_{2}, 60 \mu \mathrm{g} / \mathrm{ml}$ proteinase $\mathrm{K}, 0.45 \% \mathrm{NP} 40,0.45 \%$ Tween 20 and $0.01 \%$ gelatin) on a glass slide under a dissecting microscope. The crude lysate was transferred to a new tube. After freezing at $-80^{\circ} \mathrm{C}$ for $20 \mathrm{~min}$, the solution was incubated at $65^{\circ} \mathrm{C}$ for $70 \mathrm{~min}$ and then at $95^{\circ} \mathrm{C}$ for $15 \mathrm{~min}$ and kept at $-20^{\circ} \mathrm{C}$ until use.

PCR, cloning and sequencing. Three isolates of G. rostochiensis (Izumi, Makkari, Hokkaido), G. tabacum (Yasu, Kochi) and G. hypolysi (Obihiro, Hokkaido) were used for sequencing. The ITS regions of rDNA (ITS-rDNA) were amplified using the primers of Ferris et al. (1993). The primer sequences were 5'-CGTAACAAGGTAGCTGTAG$3^{\prime}$ and 5'-TCCTCCGCTAAATGATATG-3'. PCR was performed in a $25-\mu 1$ reaction mix containing about $4 \mu \mathrm{l}$ of crude lysate, 1 unit Takara Ex Taq ${ }^{\mathrm{TM}}$ (Takara-bio), $0.2 \mathrm{~mm}$ dNTPs, $0.2 \mu \mathrm{M}$ of each primer, and $10 \times$ Ex Taq buffer (20 $\mathrm{mM} \mathrm{Mg}^{2+}$ Plus) (Takara-bio). The PCR amplification profile consisted of $4 \mathrm{~min}$ at $94^{\circ} \mathrm{C}, 35$ cycles of $30 \mathrm{~s}$ at $94^{\circ} \mathrm{C}$, $30 \mathrm{~s}$ at $55^{\circ} \mathrm{C}$ and $30 \mathrm{~s}$ at $72^{\circ} \mathrm{C}$, followed by a final step of $5 \mathrm{~min}$ at $72^{\circ} \mathrm{C}$. Five microliters of the PCR product was analyzed by electrophoresis in a 3.0\% TAE-buffered agarose gel (Sigma Type II-A). PCR products were cloned into pCR2.1 (Invitrogen). Three clones of each isolate were used for DNA sequencing. Plasmid inserts were sequenced by dideoxynucleotide chain termination using an $\mathrm{ABI}$

Table 1. Origins of Globodera species used in this study

\begin{tabular}{|c|c|c|c|}
\hline \multirow{2}{*}{ Species } & \multicolumn{3}{|r|}{ Locality in Japan } \\
\hline & Prefecture & $\begin{array}{l}\text { City, Town or } \\
\text { Village }\end{array}$ & Area \\
\hline \multirow[t]{16}{*}{ G. rostochiensis } & Hokkaido & Bifuka & Niupu \\
\hline & & Hakodate & Furukawa, Ishikawa, Toyohara \\
\hline & & Kimobetsu & Aikawa, Fukuoka, Hiraoka, Rusan, Shiribetsu, Suzukawa \\
\hline & & Kiyosato & Kamishari-higashi, Kamishari-naka, Kamui, Kouyo \\
\hline & & Koshimizu & Mizukami \\
\hline & & Kucchan & Fujimi, Fuso, Kotohira, Midori, Mizuho, Suehiro, Takami, Takasago, Tatsumi \\
\hline & & Kyogoku & Kawanishi, Koushin, Kyogoku, Misaki \\
\hline & & Makkari & Hikari, Izumi*, Kamisato, Midorioka, Miharashi, Sakuragawa \\
\hline & & Nakashibetsu & Musa \\
\hline & & Niseko & Fujikawa, Higashiyama, Kondo, Niseko, Nishiyama, Satomi, Tomikawa \\
\hline & & Obihiro & Taisyo \\
\hline & & Rusutsu & Izumikawa, Rusutsu, Toyooka \\
\hline & & Shari & $\begin{array}{l}\text { Fuji, Ikushinakita, Ikushinaminami, Koshikawa, Misaki, Nakashari, } \\
\text { Raiun, Toyokura, Toyosato, Syuennishi }\end{array}$ \\
\hline & Aomori & Kanagi & Ashino, Shichisekino \\
\hline & & Misawa & Sabishirotai, Shiogama, Shikanaka, Shimono \\
\hline & Nagasaki & $\begin{array}{l}\text { Kazusa } \\
\text { Minamiarima }\end{array}$ & \\
\hline G. tabacum & Kochi & Yasu* & \\
\hline G. hypolysi & $\begin{array}{l}\text { Hokkaido } \\
\text { Nagasaki }\end{array}$ & Obihiro* & \\
\hline
\end{tabular}

* These three isolates were used for sequencing. 
PRIZM ready reaction kit (Applied Biosystems) in an Applied Biosystems 377 automated sequencer. DNA sequences were analyzed using DNASIS (Version 3.5) software.

RFLP. The PCR products were digested separately with each of the restriction enzymes (AfaI, AluI, FspI, HhaI, HinfI, MvaI, PmaCI and SacII). The restriction enzymes were selected on the basis of their sequence information. For six isolates of $G$. rostchiensis (Kamishari-naka, Kiyosato, Hokkaido; Obihiro, Hokkaido; Izumi, Makkari, Hokkaido; Furukawa, Hakodate, Hokkaido; Ashino, Kanagi, Aomori; Kazusa, Nagasaki), two isolates of G. hypolysi (Obihiro, Hokkaido; Nagasaki) and one isolate of G. tabacum (Yasu, Kochi), each PCR product was digested with the eight restriction enzymes. For all of the $64 \mathrm{G}$. rostochiensis isolates, each PCR product was digested with the two restriction enzymes (AluI and $F s p \mathrm{I})$. Digestion was conducted in a mixture of $5 \mu \mathrm{l}$ of the PCR product, $0.5 \mu 1$ of restriction enzyme, $1.0 \mu 1$ of $10 \times$ buffer and $4.0 \mu \mathrm{l}$ of double-distilled water. The digested DNA fragments were separated by electrophoresis on a $3.0 \%$ agarose gel, stained with ethidium bromide, and visualized on a UV transilluminator.

\section{RESULTS}

\section{Sequence analysis}

The approximate size of the ITS-rDNA PCR products was $1,000 \mathrm{bp}$ for all species (Fig. 1). The aligned ITS-rDNA sequences from the clones of $G$. rostochiensis, G. tabacum and $G$. hypolysi are shown in Fig. 2. Sequence data are available from the DDBJ/EMBL/GenBank databases under the accession numbers AB207271, AB207272 and AB207273. Sequence lengths of $G$. rostochiensis, G. tabacum and G. hypolysi were 1,001, 1,003 and $1,016 \mathrm{bp}$, respectively. Sequence divergence within the three Globodera species ranged from 3.8 to $14.7 \%$. The ITS-rDNA sequences of G. rostochiensis and G. tabacum were similar to each other (sequence similarity: 96.2\%). Sixteen nucleotide differences were observed between $G$. rostochiensis and $G$. tabacum. Sequence similarity between $G$. rostochiensis and $G$. hypolysi and that between $G$. tabacum and $G$. hypolysi were $85.2 \%$ and $85.8 \%$, respectively.

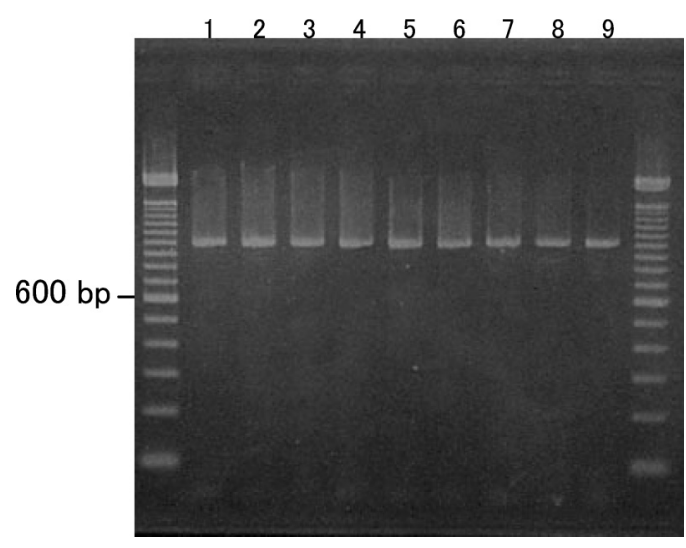

Fig. 1. Amplification of the rDNA ITS regions of Globodera species. Nematodes: 1, G. rostochiensis (Kamisharinaka, Kiyosato, Hokkaido); 2, G. rostochiensis (Obihiro, Hokkaido); 3, G. rostochiensis (Izumi, Makkari, Hokkaido); 4, G. rostochiensis (Furukawa, Hakodate, Hokkaido); 5, G. rostochiensis (Ashino, Kanagi, Aomori); 6, G. rostochiensis (Kazusa, Nagasaki); 7, G. hypolysi (Hokkaido); 8, G. hypolysi (Nagasaki); 9, G. tabacum (Kochi). The size marker on both edge lanes is a 100-bp DNA ladder.

\section{RFLP analysis of the ITS region}

AfaI, AluI, FspI, HhaI, HinfI, MvaI, PmaCI and SacII cut the PCR products into several fragments in at least one Globodera species used. The restriction patterns are shown in Fig. 3, and the fragment sizes based on sequence data except for the HhaIdigested pattern are shown in Table 2. In the case of $H h a \mathrm{I}$, the fragment sizes based on sequence data disagree with the sizes of fragments separated by electrophoresis. AluI cut the PCR products of the three Globodera species and provided species-specific restriction patterns. PmaCI cut the PCR products of G. rostochiensis and G. tabacum and provided species-specific restriction patterns. PmaCI did not cut the PCR products of G. hypolysi. Therefore, the three species were easily discriminated. FspI cut only the PCR product of G. rostochiensis into two fragments. The FspI-digested pattern distinguished G. rostochiensis from the other Globodera species. SacII cut only the PCR product of G. tabacum into two fragments. The MvaI-digested pattern showed polymorphism between the G. rostochiensis isolates and also indicated the occurrence of heterogeneity in the ITS regions. As for the five restriction enzymes, AfaI, HhaI, HinfI, $P m a \mathrm{CI}$ and SacII, intra-specific variation was not found among the six isolates of $G$. rostochiensis examined by PCR-RFLP analysis. Moreover, as for the two restriction enzymes, AluI and FspI, no 


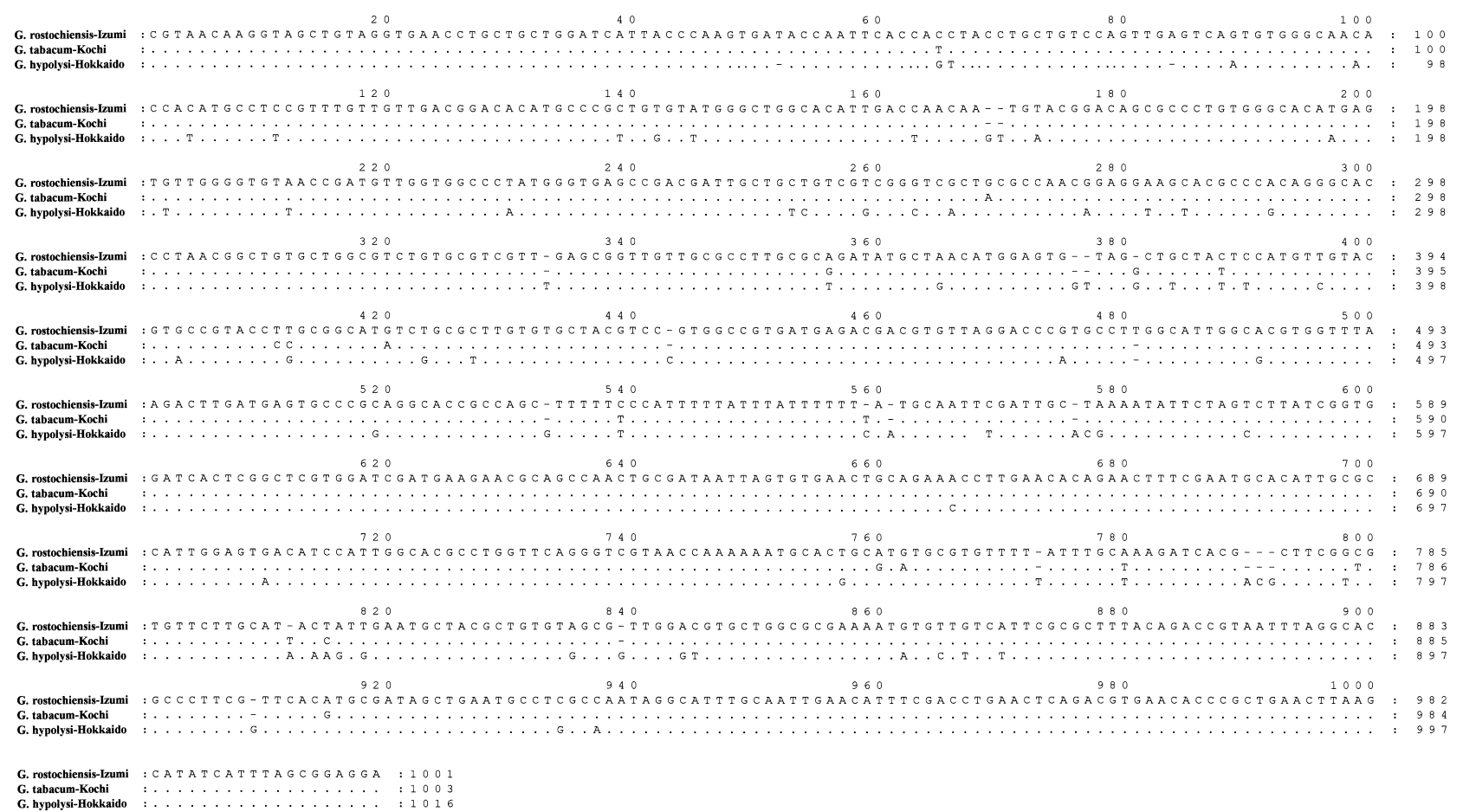

Fig. 2. Sequences of ITS1, 5.8S and ITS2 and partial sequences of 18S and 26S gene regions of rDNA from Globodera rostochiensis (Izumi, Makkari, Hokkaido), G. tabacum (Kochi) and G. hypolysi (Hokkaido). Sequence data are available in the DDBJ/EMBL/GenBank databases under the accession numbers AB207271, AB207272 and AB207273.

intra-specific variation in the restriction patterns was found among any of the G. rostochiensis isolates examined in this study.

\section{DISCUSSION}

The potato cyst nematode (G. rostochiensis) is a pest of worldwide importance, causing serious damage to potato crops. In Japan, seed-potato production is not allowed in infected fields even if other production conditions are favorable. In 2003, G. rostochiensis was found in the Tokachi region of Hokkaido, which is the best production area for seed-potatoes in Japan. The species G. hypolysi, collected from Artemisia princeps in potato fields, was described by Ogawa et al. (1983). G. hypolysi parasitic Asteraceae may assume importance even though they cause no economic damage themselves. If their cysts are found in a field for seedpotatoes and are mistakenly identified as those of the potato cyst nematode, no seed production is allowed in the infected field. The occurrence of $G$. tabacum was recorded for the first time in Japan in 1998.

The purpose of the present study was to obtain
PCR-RFLP profiles and information on the exact sizes of the restriction fragments for the three Globodera species. As for the two restriction enzymes, $A l u \mathrm{I}$ and $F s p \mathrm{I}$, no intra-specific variation in the restriction patterns was found among any of the $G$. rostochiensis examined in this study. The present work confirmed that PCR-RFLP enables clear differentiation of the three Globodera species. Amplification and analysis of ITS-rDNA have many advantages. The rapidity with which the profiles can be obtained and the clarity of the results allow for the easy identification of species. Sequence information of ITS-rDNA is useful for comparison with published sequence data. The sequences determined in this study were compared with previously published sequences for G. rostochiensis (Gr-Ja1) and G. tabacum solanacearum (Gt-Sol1) (Subbotin et al., 2000). G. rostochiensis (Izumi, Makkari, Hokkaido) and Gr-Ja1 were very similar (99.6\%), and G. tabacum (Yasu, Kochi) and Gt-Soll were also very similar to each other $(99.7 \%)$. Three nucleotide differences were observed between G. rostochiensis (Izumi, Makkari, Hokkaido) and Gr-Ja1, and two nucleotide differences were observed between G. tabacum (Yasu, Kochi) and Gt-Soll. 

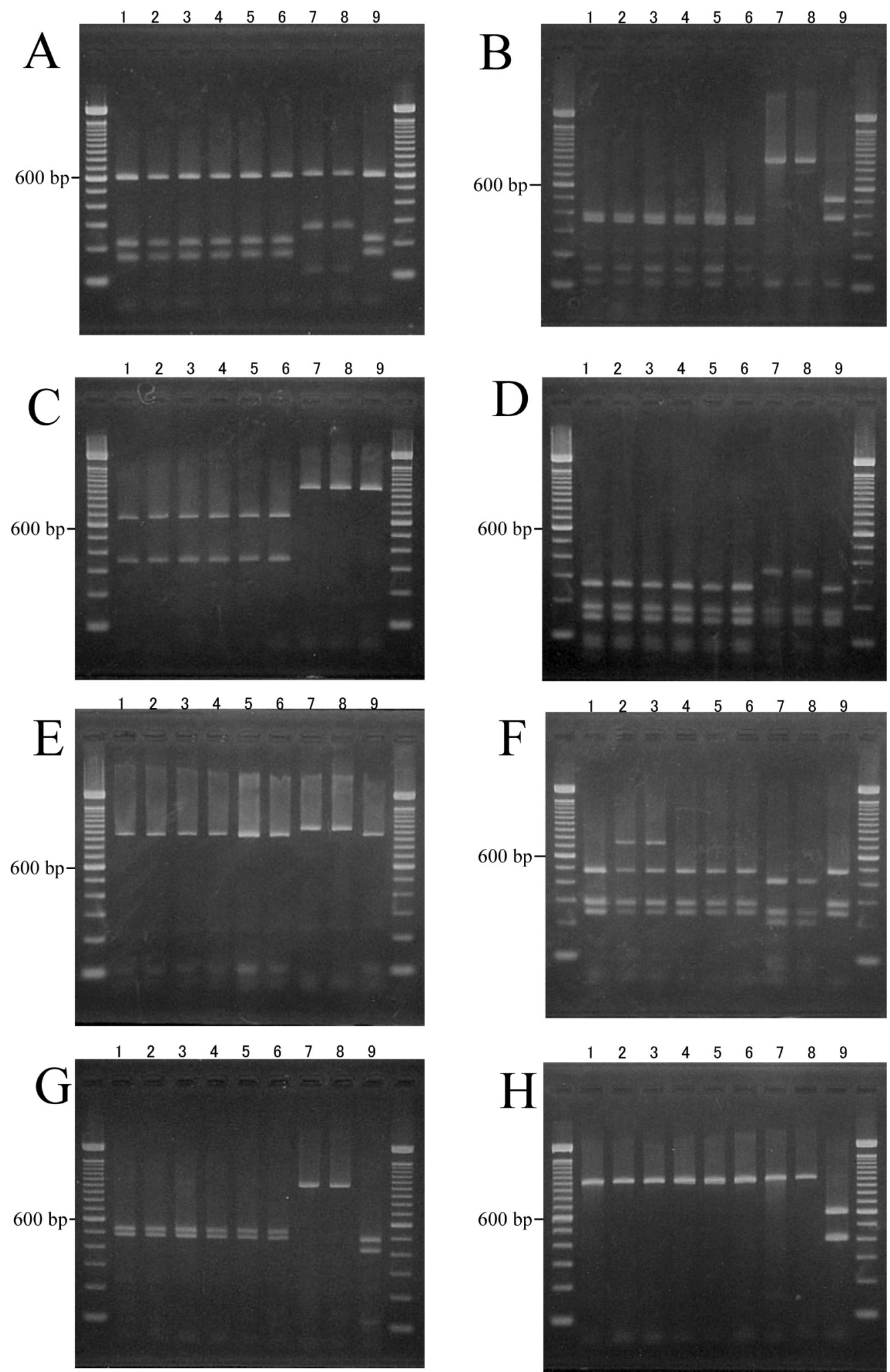

Fig. 3. Restriction fragments of the amplified ITS regions of the rDNA of Globodera species. Endonuclease: A, AfaI; B, AluI; C, FspI; D, HhaI; E, HinfI; F, MvaI; G, PmaCI; H, SacII. Nematodes: 1, G. rostochiensis (Kamishari-naka, Kiyosato, Hokkaido); 2, G. rostochiensis (Obihiro, Hokkaido); 3, G. rostochiensis (Izumi, Makkari, Hokkaido); 4, G. rostochiensis (Furukawa, Hakodate, Hokkaido); 5, G. rostochiensis (Ashino, Kanagi, Aomori); 6, G. rostochiensis (Kazusa, Nagasaki); 7, G. hypolysi (Hokkaido); 8, G. hypolysi (Nagasaki); 9, G. tabacum (Kochi). The size marker on both edge lanes is a 100-bp DNA ladder. 


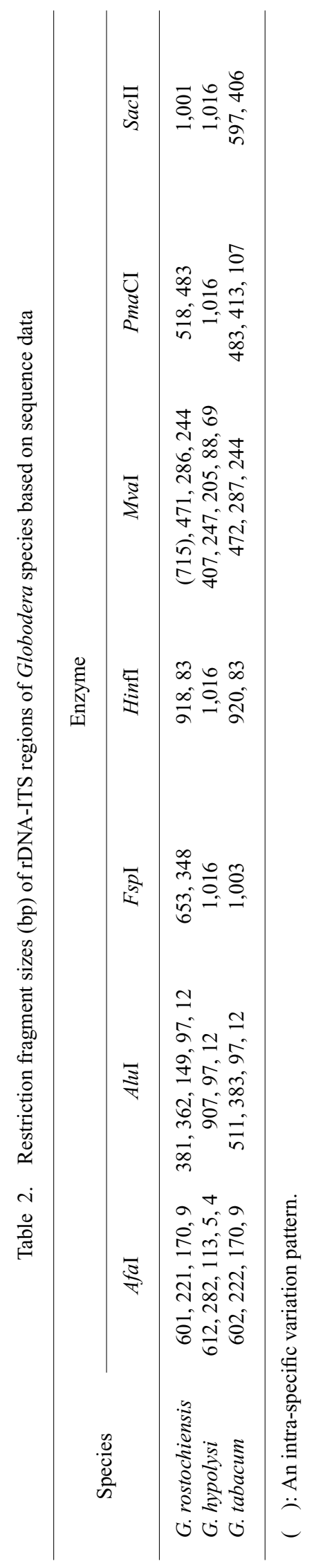

These findings are in good agreement with results of morphological and morphometrical studies. GrJa1 and Gt-Soll were estimated to show speciesspecific patterns by digesting the amplified products with $A l u \mathrm{I}$ or PmaCI based on the sequence information. Sequence divergences of the $G$. tabacum complex were insufficient to distinguish these subspecies.

Orui (1997) reported the distinction of G. rostochiensis and four Heterodera species from Japan. We have reported here the distinction of three Globodera species. Twelve species of cyst-forming nematodes (Heterodera, Globodera, Cactodera and Afenestrata), including hitherto undescribed species, have been found in Japan. Cyst-forming nematodes include important agricultural pests, such as G. pallida and H. schachtii, which have not been found in Japan. Subbotin et al. (1999) reported that $A f a \mathrm{I}, A l u \mathrm{I}, B s p 143 \mathrm{I}$ and HinfI generated polymorphism and clearly distinguished between G. rostochiensis and G. pallida. RFLP profiles and the sequences of ITS regions are particularly useful for determining the identities of quarantine nematodes. It is necessary to obtain RFLP profiles in the ITS-rDNA of more species. The MvaI digestion pattern showed polymorphism among the G. rostochiensis populations in this study. Subbotin et al. (2000) reported that StyI generated polymorphism among G. rostochiensis isolates. Sequence variations in ITS-rDNA in populations of G. rostchiensis and G. pallida have been studied (Thiéry and Mugniéry 1996; Blok et al., 1998). Furthermore, the range of intra-specific variation should be studied to apply PCR-RFLP as a routine tool for the identification of cyst-forming nematodes in any institution.

\section{ACNOWLEDGEMENTS}

The first author gratefully acknowledges financial support from an OECD Fellowship and the Akiyama Foundation. The authors thank K. Hirata (Yokohama Plant Protection Station), M. Shimomoto (Kochi Agriculture Research Center), Y. Yamada (Snow Brand Seed Co. Ltd.), and Y. Kimura and T. Fujimura (Aomori Prefecture Agricultural and Forestry Research Center) for supplying nematode populations, and V. C. Blok (Scottish Crop Research Institute) for her valuable advice.

\section{REFERENCES}

Blok, V. C., G. Malloch, B. Harrower, M. S. Phillips and T. C. Vrain (1998) Intraspecific variation in ribosomal DNA in populations of the potato cyst nematode Globodera pallida. J. Nematol. 30: 262-274. 
Ferris, V. R., J. M. Ferris and J. Faghihi (1993) Variation in spacer ribosomal DNA in some cyst-forming species of plant parasitic nematodes. Fundam. Appl. Nematol. 16: 177-184.

Iwahori, H., K. Tsuda, N. Kanzaki, K. Izui and K. Futai (1998) PCR-RFLP and sequencing analysis of robosomal DNA of Bursaphelenchus nematodes related to pine wilt disease. Fundam. Appl. Nematol. 21: 655-666.

Mizukubo. T., Y. Orui, K. Hanada and Z. Sano (2003) Microevolutionary trend in Pratylenchus coffeae sensu stricto (Nematode: Pratylenchidae): the diversity in PCRRFLP phenotype, compatibility on host plants and reproductive segregation. Jpn. J. Nematol. 33: 57-76.

Nakasuga, T. and K. Nakasono (1996) New finding of potato cyst nematode, Globodera rostochiensis from potato fields in Nagasaki Prefecture, Japan. Jpn. J. Nematol. 26: 42-43 (in Japanese).

Ogawa, Y., Y. Oshima and M. Ichinohe (1983) Globodera hypoysi n. sp., a mugwort cyst nematode from Japan. Jpn. J. Nematol. 12: 41-46.

Orui, Y. (1997) Discrimination of Globodera rostochiensis and four Heterodera species (Nematode: Heteroderidae) by PCR-RFLP Analysis. Jpn. J. Nematol. 27: 67-75 (in Japanese).

Orui, Y. and T. Mizukubo (1999) Discrimination of seven Pratylenchus species (Nematode: Pratylenchidae) in Japan by PCR-RFLP analysis. Appl. Entomol. Zool. 34: 205-211.

Subbotin, S. A., P. D. Halford, A. Warry and R. N. Perry (1999) Identification of populations of potato cyst ne- matodes from Russia using protein electrophoresis, rDNA-RFLP and RAPDs. Russ. J. Nematol. 7: 57-63.

Subbotin, S. A., P. D. Halford, A. Warry and R. N. Perry (2000) Variations in ribosomal DNA sequences and phylogeny of Globodera parasitising solaneceous plants. Nematology 2: 591-604.

Sumiya, T., K. Hirata and T. Yaegashi (2002) Identification of three Globodera species in Japan by using isoelectric focusing method. Res. Bull. Plant Protection Service Japan 38: 49-51.

Thiéry, N. and D. Mugniéry (1996) Interspecific rDNA restriction fragment length polymorphism in Globodera species, parasites of Solanaceous plants. Fundam. Appl. Nematol. 19: 471-479.

Uehara, T., T. Mizukubo, A. Kushida and Y. Momota (1998) Identification of Pratylenchus coffeae and P. loosi using specific primers for PCR amplification of ribosomal DNA. Nematologica 44: 357-368.

Vrain, T. C., D. A. Wakarchuk, A. C. Levesque and R. I. Hamilton (1992) Intraspecific rDNA restriction fragment length polymorphism in the Xiphinema americanum group. Fundam. Appl. Nematol. 15: 563-573.

Yamada, E. and S. Takakura (2003) Discovery of Globodera hypolysi from mugwort, Artemisia montana, in the east of Hokkaido. Jpn. J. Nematol. 33: 14-22 (in Japanese).

Yamada, E., S. Takakura and H. Tezuka (1972) On the occurrence of the potato cyst nematode, Heterodera rostochiensis Wollenweber in Hokkaido, Japan. Jpn. J. Nematol. 2: 12-15. 